\title{
Presentations of the Injection Drug Users in an Emergency of a Surgical Unit of a Tertiary Care Hospital of Pakistan
}

\author{
Muhammad Ayyub Anjum¹, Ayesha Shaukat²
}

1,2Department of Surgery, West Surgical Ward, King Edward Medical University /Mayo Hospital, Lahore-Pakistan

\begin{abstract}
Background: Drug Abuse is a growing concern in whole world and in Pakistan we are seeing increasing number of people using drugs. There is growing shift towards injection drug abuse. Young generation is falling victim to Injection drug abuse which not only poses serious medical concerns but also present as life threatening emergencies on surgical floor. We have been seeing increasing number of people coming with ruptured pseudo aneurysms on surgical floor with resultant life-threatening hemorrhage for the patient and putting healthcare providers too at risk of getting various infectious diseases like HIV. Objective: The aim was to study the different presentations of injection drug users in surgical emergency and assess the seroprevalence of viral infections and factors associated with injection drug use. Study Design: Retrospective study. Settings: The study was conducted in west surgical ward King Edward medical university Lahore-Pakistan. Duration: Six months from 01-01-2019 to 01-06-2019. Methodology: data of all the injection drug users presenting in surgical emergency was collected. This comprised their sociodemographic profile, presenting disease and blood tests for HIV, Hepatitis B and Hepatitis C virus infections. Results: The mean age of patients was 31 years. Majority (47 $\%$ ) belonged to Lahore, $21 \%$ from Gujranwala and $16 \%$ from Sialkot. Majority $(89.5 \%)$ had poor socioeconomic status. All patients were males. Majority $(63.2 \%$ ) were single and illiterate $(73.7 \%)$. In all the patients route of injection was femoral. Regarding viral serology $84.2 \%$ patients were HIV positive ,15.8\% patients were positive for both HIV and HCV. Patients presented with three surgical problems; Ruptured Femoral pseudoanuerysms $(42.1 \%$, $n=8)$, Abscesses $(31.6 \%, n=6)$, Fournier's gangrene $(26.3 \%, n=5)$. In all femoral Psuedoanuerysms external iliac artery was ligated. Abscesses were incised and drained. Fournier's gangrene was debrided with exposure of the testes. Conclusion: The Government should control illegal drug usage, educate the masses through print and electronic media to raise awareness of growing epidemic of HIV and to provide resources to made dedicated centers for surgical emergencies of these patients.
\end{abstract}

Keywords: Injection drug, Surgical emergency, Seroprevalence of viral infections.

Corresponding Author

Submitted for Publication: 05-05-2020

Accepted for Publication: 15-08-2020

Dr. Muhammad Ayyub Anjum, Trainee Registrar, West Surgical Ward, King Edward Medical University /Mayo Hospital, Lahore-Pakistan

Email: drayyub212@yahoo.com

Citation: Anjum MA, Shaukat A. Presentations of the Injection Drug Users in an Emergency of a Surgical Unit of a Tertiary Care Hospital of

Pakistan. APMC 2020;14(3):205-8.

DOI: $10.29054 / A P M C / 2020.924$

\section{INTRODUCTION}

Humans Have been using drugs since prehistoric times. Egyptians used wine in 4000 B.C. In china Medicinal use of marijuana was common around 3000 B.C. Drug abuse is a patterned use of a substance (drug) in which the user consumes the substance in amounts or with methods which are harmful to themselves or others. ${ }^{1}$

Drug abuse is a major problem all over the world. According to world health organization about 31 million people in the world use drugs and almost 11 million people are injection drug abusers. Among Injection drug abusers 1.3 million are HIV positive, 5.5 million are hepatitis $C$ positive, and 1 million have both HIV and hepatitis C. ${ }^{2}$ In Pakistan 430,000 people, which constitute $0.4 \%$ of population, are Injection drug abusers. $73 \%$ of these Injection drug abusers in Pakistan share syringes. ${ }^{3}$

Initiation of injection drug use is associated with age, marital status, low income, illiteracy, juvenile arrest history, earlier age of drug initiation, type of drug used first time, multiple sex partners, earlier age of sexual experience and casual sex partners. Poor knowledge about the prevalence of HIV increased the likelihood of transition to injection drug use from oral drug use. ${ }^{4}$ Mean age of Injection drug use is 38 years and in a study conducted in Lahore Pakistan, All were males (100\%). $75 \%$ of Injection drug abusers were unmarried, illiterate (52.5\%) and unemployed (77.5\%). $95 \%$ belonged to lower socioeconomic group. $58 \%$ of the time groin is the most common site for Injection drug use. Heroin is the most common drug used among population in Pakistan. This practice has lead to increased incidence of viral infections like HCV, HBV and HIV. ${ }^{5}$

Local complications in the groin are local cellulitis, abscess formation and femoral pseudoanuerysms. Pseudoanuerysms lack the well-defined wall and are just lined by granulation tissue. They carry the risk of spontaneous rupture and lifethreatening hemorrhage and is a surgical emergency. ${ }^{6}$ These cases require aggressive resuscitation and ligation of the vessel is the only option in most of the cases. These are high risk cases to operate on a General Surgical floor and standard precautions are required for health care professionals operating and managing such cases. ${ }^{7}$ There is a need for eye protection, double gloving, specialized protective clothing while operating such cases leading to high demand of hospital resources and increased risk of transmission of HIV, HCV and HBV to health professionals. ${ }^{8,9}$ 
This study is conducted for injection drug abusers who presented in emergency of a tertiary care hospital of Lahore and will improve the existing knowledge on the subject and will guide healthcare authorities to allocate resources in creating awareness among general population regarding hazards of injection drug use and also the hospital authorities to allocate resources for safety of health care professionals managing such cases, separate operation rooms and kits and proper measures to ensure sterilization after the procedure.

\section{METHODOLOGY}

Study Design: Retrospective study.

Settings: Surgical emergency ward King Edward Medical University Lahore-Pakistan.

Duration: 06 months from January 1, 2019 to June 30, 2019.

Sample Technique: Consecutive non-random sampling.

Sample Size: A total of 18 patients presented on our surgical floor during this period.

Inclusion Criteria: All patients with history of Intravenous Drug Abuse, patients of any gender and age and patients presenting in Emergency only was included.

Exclusion Criteria: No Patient was excluded from the study who had history of IV drug abuse and presented in surgical emergency.

Data Collection Procedure: A detailed History and examination were carried out for each patient. Screening for Hepatitis B virus, Hepatitis C virus and Human Immune Deficiency virus was done in each patient. With proper preoperative preparation and safety measures each patient was managed for his surgical disease. Data collection included age, presenting disease, socioeconomic status, marital status, gender. Data was tabulated and analyzed with SPSS v 20.0.

\section{RESULTS}

In our study, the mean age of patients presenting in our emergency was 31 years. We made groups of patients regarding age as shown in figure 1 . The majority of patients presenting belonged to age group 21 to 40 years. Injection drug users belonged to younger age group. $47 \%$ of patients presenting in our hospital belonged to Lahore, $21 \%$ from Gujranwala and $16 \%$ from Sialkot as shown in figure 2. Majority $(89.5 \%)$ of the patients belonged had poor socioeconomic status (figure 3) and all patients were males (table 4). $63.2 \%$ patients were single and never married in their life, remaining $36.8 \%$ patients were married (Figure 4). In all the patients route of injection was femoral. Regarding viral serology $84.2 \%$ patients were HIV positive remaining $15.8 \%$ patients were both HIV and HCV positive (figure 05). $73.7 \%$ of the patients were illiterate and never went to school in their life (figure 06). Patients presented with three surgical problems; Ruptured Femoral pseudoanuerysms $(42.1 \%, \mathrm{n}=8)$, Abscesses $(31.6 \%, \mathrm{n}=6)$, Fournier's gangrene $(26.3 \%, n=5)$ table 08 . All femoral pseudoanuerysms had to be ligated proximally and in all patients external iliac artery was ligated. Abscesses were incised and drained and consisted of abscesses on various parts of body. Fournier's gangrene was debrided with exposure of the testes.

Table 1: Gender

\begin{tabular}{|c|c|c|c|c|}
\hline & Frequency & Percent & $\begin{array}{c}\text { Valid } \\
\text { Percent }\end{array}$ & $\begin{array}{c}\text { Cumulative } \\
\text { Percent }\end{array}$ \\
\hline Male & 19 & 100.0 & 100.0 & 100.0 \\
\hline
\end{tabular}

Table 2: Diagnosis

\begin{tabular}{|c|c|c|c|c|}
\hline Valid & Frequency & Percent & $\begin{array}{c}\text { Valid } \\
\text { Percent }\end{array}$ & $\begin{array}{c}\text { Cumulative } \\
\text { Percent }\end{array}$ \\
\hline Fournier's gangrene & 5 & 26.3 & 26.3 & 26.3 \\
\hline Abscess & 6 & 31.6 & 31.6 & 57.9 \\
\hline Pseudo aneurysm & 8 & 42.1 & 42.1 & 100.0 \\
\hline Total & 19 & 100.0 & 100.0 & \\
\hline
\end{tabular}

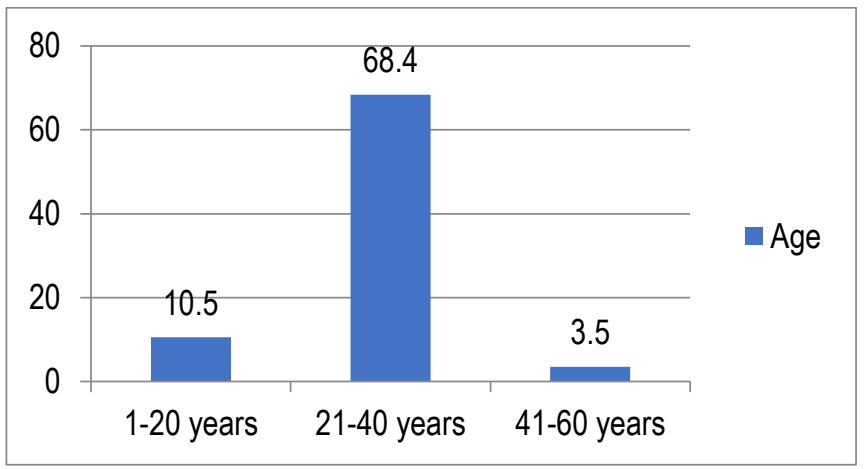

Figure 1: Age

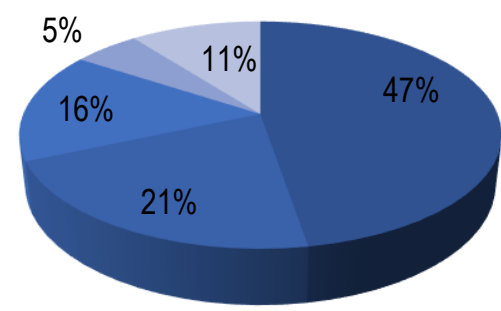

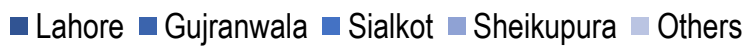

Figure 2: Address

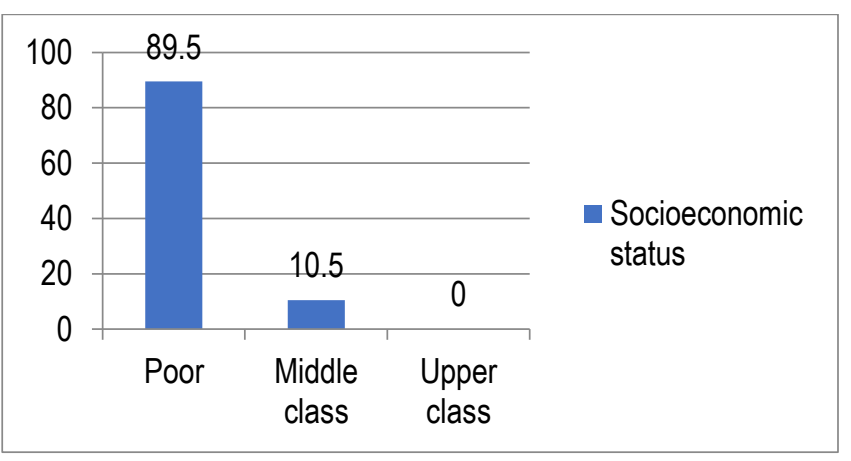

Figure 3: Socioeconomic status 


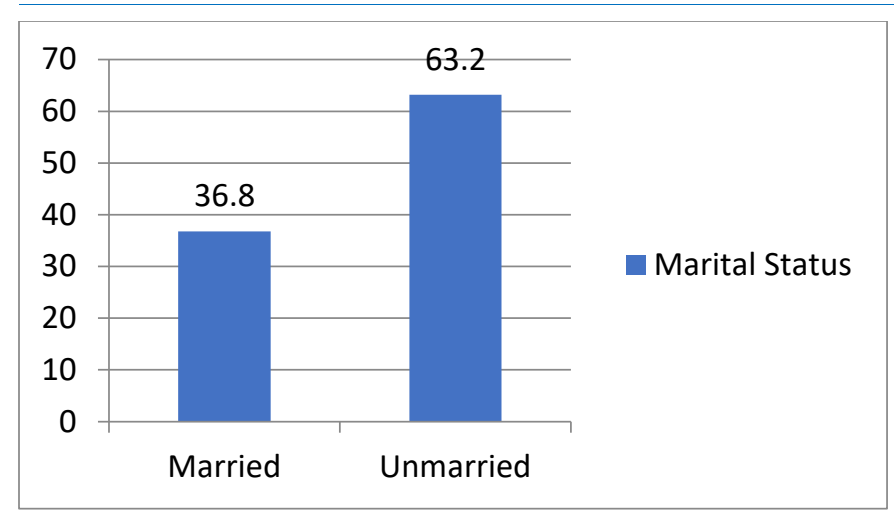

Figure: 4 Marital Status

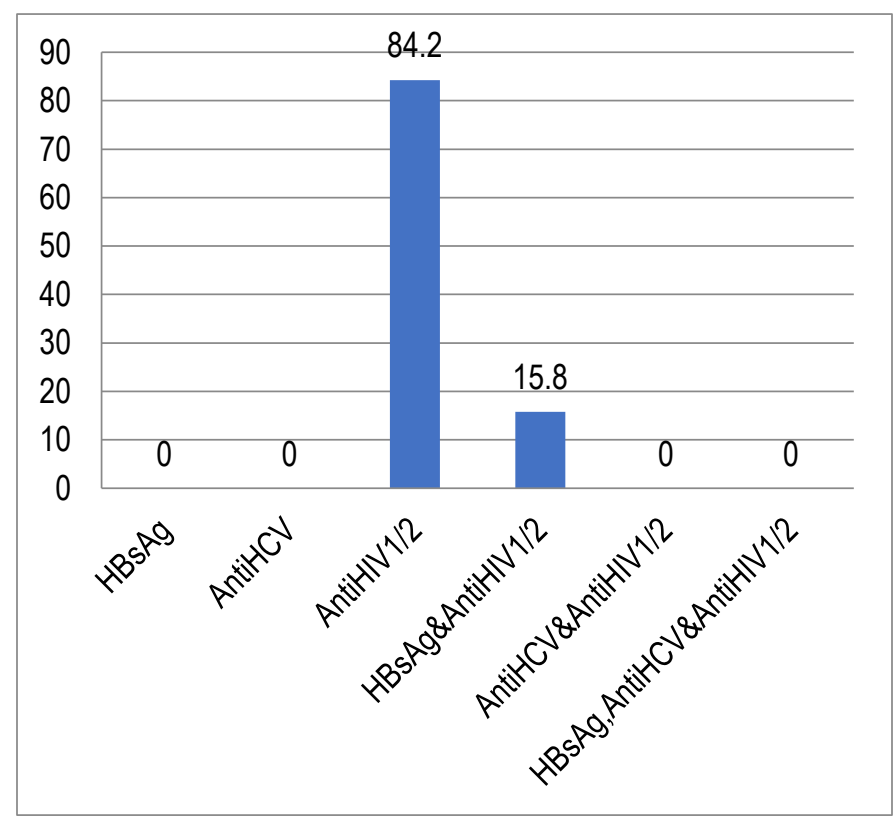

Figure: 5 Serology

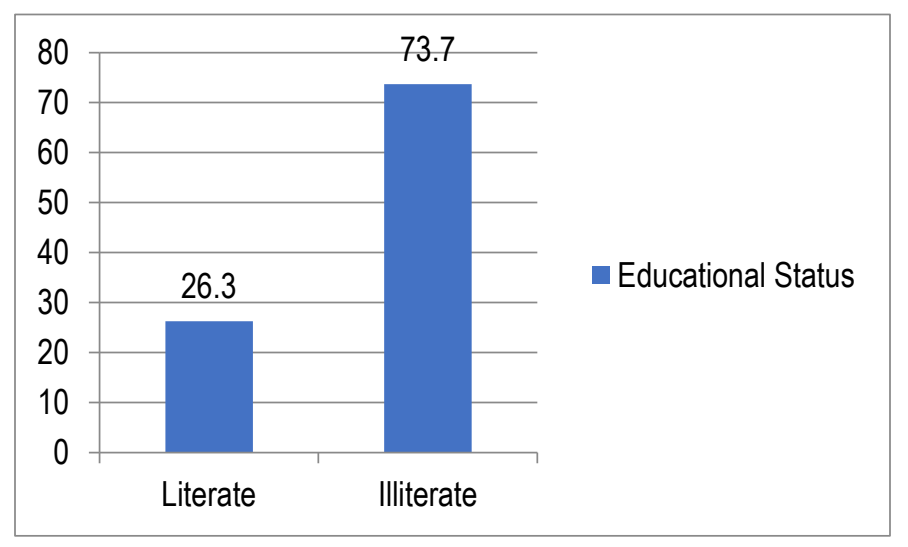

Figure: 6 Educational Status

\section{DISCUSSION}

Mean age of presentation in our study was 31 years in our study with majority of the patients ranging in age from 20 to 40 years. Syed Hamad Rasool et al. in a study of 2009 conducted in our hospital reported mean age of 38 with age range of 24 to 54 years. ${ }^{5}$ This indicates a significant shift to younger age group of
Injection drug use just in 10 years time. Syed Ali Raza et al in their cross-sectional survey of 2013-14 in five major cities in Pakistan showed mean age of 33.3 years with age ranging from 16 to 70 years 4 .A study conducted in India showed mean age of 32.7 years with age ranging from $18-72$ years. ${ }^{10}$

All patients presented to us were males and majority of patients belonged to poor socioeconomic class. $73 \%$ of people in our study were illiterate and never went to school in their life while another study conducted in Lahore showed $52 \%$ of people were illiterate. $^{5}$

In our study $63.2 \%$ of patients were single and never married in their life. A study conducted in Lahore showed $75 \%$ of the injection drug users were single and never married in their life. ${ }^{5}$ All of our patients used groin area for injecting drugs since the start of their addiction. In other studies, conducted in Pakistan injection drug use in groin area $80 \%{ }^{5}$. An Iranian study however demonstrated around $30 \%$ of patients used groin for injecting drugs. ${ }^{11} \mathrm{~A}$ study conducted in Australia demonstrated that only $6 \%$ of their injection drug users used groin as the injecting site. ${ }^{12}$ The most common site for drug injection in studies conducted in other countries was forearm.11,12 Majority of patients use heroin. ${ }^{5}$

$82.2 \%$ of Injection drug users presenting to our surgical emergency tested positive for HIV and the remaining $15.8 \%$ were positive for both HIV and HCV. None of the patients in our study was positive for HBV. Similar study in Lahore showed 10 $\%$ injection drug users were HIV positive, $35 \%$ HBV positive and $42 \%$ positive for $\mathrm{HCV}$. The study was conducted in general population of Lahore. ${ }^{5}$ This also shows that injection drug users who present with surgical complication are more likely to have been addicts for longer and have shared syringes and overwhelming percentage of them being HIV positive in surgical emergency poses serious risks to other patients and staff and burden on hospital resources. Studies have shown that $42 \%$ of injection drug users in Ukraine are HIV positive, $37 \%$ injection drug users in Russia are HIV positive, $12 \%$ Injection drug users in China are HIV Positive. In the USA 9\% were HIV positive. ${ }^{13}$

Patients presented with three surgical problems in our emergency; Ruptured Femoral pseudoanuerysms (42.1\%, $n=8)$, Abscesses (31.6\%, $n=6)$, Fournier's gangrene $(26.3 \%$, $n=5)$. All femoral pseudoanuerysms external iliac artery was ligated. None of the patient had to go for amputation after vessel ligation. Abscesses were incised and drained. Fournier's gangrenes were debrided with exposure of the testes. Patient with Pseudoanuerysms presented in Hypovolemic Shock due to rupture of pseudoanuerysms.

A review article which covered data from 1966 to 2005 on arterial consequences of injection drug use demonstrated that pseudoanuerysms develop more commonly in femoral artery and they pose a serious risk due to their propensity to rupture and cause life threatening hemorrhage. Vessel ligation was the most effective treatment in this regard and most of the patients usually does not need amputation. Revascularization proves high risk as regard to risk of death due to pulmonary embolism and rebleeding. ${ }^{6}$ 


\section{CONCLUSION}

The increasing trend of injection drug use in our society is combined with low literacy rate and no awareness to associated health risks is posing as a serious health problem. Patients who present in surgical emergency pose health risks to doctors, paramedical staffs and other patients and increase burden on the hospital resources. There is an impending need to devise plans for awareness, education and provide rehabilitative services to these patients . separate operation theatres should be allocated to operate on these patients with proper protective gear for health care providers. Only by the combined efforts of the government and society we can stop this growing epidemic in our society.

\section{LIMITATIONS}

The major limitation we faced while conducting this study was lack of any online software in tertiary care teaching hospitals where we can keep record of injection drug abusers which can help to identify people timely and also help them in follow up and rehabilitation. The patients either don't admit or feel ashamed to admit that they abuse drugs so finding patients without pathognomic signs of drug abuse was a hard task.

\section{SUGGESTIONS / RECOMMENDATIONS}

The Government should include local law enforcement and political management to limit the sale and purchase of the explicit drugs in the market and use effective electronic, print and social media usage to raise the awareness of the hazards of the drug abuse. There should be an online software maintaining all the data of Injection drug abusers presenting in the hospital so that they can be followed up and can be helped with rehabilitation.

\section{CONFLICT OF INTEREST / DISCLOSURE}

We have no conflict of interests.

\section{ACKNOWLEDGEMENTS}

We Acknowledge and thank our all residents house surgeons and faculty of the surgical department at KEMU/ Mayo Hospital Lahore and especially of west surgical ward for their continuous support and motivation in conducting this study in our unit.

\section{REFERENCES}

1. Substance abuse - Wikipedia, the free encyclopedia. Available online at: http://en.wikipedia.org/wiki/Substance_abuse

2. World health Organization statistics. Available online at: https://www.who.int/substance_abuse/facts/en/ 2018

3. Ministry of interior and narcotics control division Government of Pakistan, united nations office on drugs and crime. Drug use in Pakistan 2013.

4. Raza MA, Sherwani RHK, Kamal S, Aftab M, Irfan M. Correlates of injection drug initiation among drug users in Punjab Pakistan. PJMHS. 2016;10(4):1135-9.

5. Rasool SH, Arif M, Ahmed M. Sociodemographic Profile of Intravenous Drug Users in Lahore - A Retrospective Study. PJMHS. 2014;8(2):145-56.
6. Coughlin PA, Mavor AID. Arterial Consequences of Recreational Drug Use. Eur J Vasc Endovasc Surg. 2006;32(4):389-96.

7. Judd A, Hickman M, Jones S, Mcdonald T, Parry JV, Stimson GV et al. Incidence of hepatitis $C$ virus and HIV among new injecting drug users in London: prospective cohort study. BMJ. 2005;330(7481):24-5.

8. Berridge DC, Lees TA, Chamberlain J, Jones NA. Eye protection for the vascular surgeon. Br J Surg. 1993;80(11):1379-80.

9. Berridge DC, Starky G, Jones NA, Chamberlain J. A randomized controlled trial of double-versus single-gloving in vascular surgery. J R Coll Surg Edinb. 1998;43(1):9-10.

10. Saini ns, Luther A, Mahajan A, Josep A. infected pseudoaneurysms in intravenous drug abusers: Ligation or reconstruction. Int J Appl Basic Med Res. 2014;4(1):23-6.

11. Karimi M, Ghaheri H, Assari S. etal. Drug injection to sites other than arm: A study of Iranian heroin injectors. Front Psychiatry. 2014;5:23.

12. Darke S, Ross J, Kaye S. Physical injection sites among injection drug users in Sydney, Australia. Drug Alcohol Depend. 2001;62(1):77-82.

13. Don C. Jarlais D, Arasteh K, Semaan S, Wood E. HIV among Injection drug users: current epidemiology, biomarkers, respondent driven sampling and supervised injection facilities. Curr Opin HIV AIDS. 2009;4(4):308-13.

\section{AUTHORSHIP CONTRIBUTION}

\begin{tabular}{|l|l|}
\hline Dr. Muhammad Ayyub Anjum & \\
Trainee Registrar, West & Data Collection \\
Surgical Ward, King Edward & \\
Medical University /Mayo & \\
Hospital Lahore Pakistan & \\
\hline Dr. Ayesha Shaukat & \\
Professor of Surgery, West & Manuscript Writing, Critical Review \\
Surgical Ward, King Edward & \\
Medical University/ Mayo & \\
Hospital Lahore Pakistan & \\
\hline
\end{tabular}

